\title{
Saúde mental e qualidade de vida de pais de pessoas com TEA durante a pandemia COVID-19: uma revisão narrativa
}

\author{
Mental health and quality of life of \\ parents of people with ASD during \\ COVID-19: literature review
}

\section{Aracelles Alvarenga Medrado' (1) Rafael Cabral Campos ${ }^{2}$ (1) Gustavo Marcelino Siquara3 3 Milena Pereira Pondé 4 (1)}

\begin{abstract}
'Autora para correspondência. Escola Bahiana de Medicina e Saúde Pública (Salvador). Bahia, Brasil. aracellesmedrado17.1@bahiana.edu.br 2-4Escola Bahiana de Medicina e Saúde Pública (Salvador). Bahia, Brasil. rafaelcampos17.2@bahiana.edu.br, gustavosiquara@bahiana.edu.br, milenaponde@bahiana.edu.br
\end{abstract}

\begin{abstract}
RESUMO | INTRODUÇÃo: Em dezembro de 2019 surgiram, na China, os primeiros caso de COVID-19, uma doença infectocontagiosa que, em poucos meses, evoluiu para o estado de pandemia. Foram adotadas medidas de distanciamento social, que podem gerar impactos psicológicos negativos para a população. O impacto pode ser maior para os pais de indivíduos com TEA, já que as demandas de cuidados dos filhos passam para os genitores em tempo integral. Soma-se ainda o fato de o trabalho dos pais em home-office e as demandas de trabaIho doméstico. OBJETIVO: Revisar estudos sobre a saúde mental e/ou qualidade de vida de pais durante a pandemia. METODOLOGIA: Tratase de uma revisão narrativa da literatura buscando-se artigos nas bases PubMed/Medline, Scielo (Scientific Electronic Library Online), Google Scholar, Portal BVS (Biblioteca Virtual em Saúde), Periódicos Capes (Coordenação de Aperfeiçoamento de Pessoal de Nível Superior). Foram selecionados 12 artigos de estudos qualitativos transversais a partir das bases de dados. RESULTADOS: A avaliação dos artigos mostra que os pais de indivíduos com TEA tendem a estar mais ansiosos, estressados e depressivos nesse período devido a sobrecargas físicas e emocionais. Além disso, eles apresentaram pior qualidade de vida neste período quando comparado a pais de crianças com outros transtornos e com desenvolvimento típico. CONCLUSÃO: O desenvolvimento de mais estudos sobre o tema é necessário, pois com maior embasamento científico é possível planejar um cuidado mais direcionado. 0 apoio virtual de profissionais e outros pais podem minimizar o impacto da pandemia sobre essa população. Entretanto, a diversidade de fatores sociais, econômicos e culturais envolvidos nessa questão requer mobilização multisetorial.
\end{abstract}

PALAVRAS-CHAVE: Transtorno do espectro autista. Pais. Saúde Mental. Qualidade de Vida. COVID19.
ABSTRACT | INTRODUCTION: In December 2019, the first cases of COVID-19, an infectious-contagious disease that evolved into a pandemic state, emerged in China. Social distancing measures were adopted, which can generate negative psychological impacts for the population. The impact may be greater for the parents of individuals with ASD, as the demands of the children's care are passed on to the parents on a full-time basis. In addition, the fact that parents work from home and the demands of domestic work is added. OBJECTIVE: To review studies on the mental health and/or quality of life of parents during the pandemic. METHODOLOGY: This is a narrative review of the literature looking for articles based on PubMed/Medline, Scielo (Scientific Electronic Library Online), Google Scholar, BVS Portal (Virtual Health Library), Capes Journals (Coordination for the Improvement of Health Personnel Upper level). 12 articles from qualitative crosssectional studies were selected from the databases. RESULTS: The evaluation of the articles shows that the parents of individuals with ASD tend to be more anxious, stressed, and depressed in this period due to physical and emotional overload. In addition, they had a worse quality of life in this period when compared to parents of children with other disorders and with typical development. CONCLUSION: The development of more studies on the theme is necessary because it is possible to plan more targeted care with a greater scientific basis. The virtual support of professionals and other parents can minimize the impact of the pandemic on this population. However, the diversity of social, economic, and cultural factors involved in this issue requires multisectoral mobilization.

KEYWORDS: Autism spectrum disorder. Parents. Mental health. Quality of life. COVID-19. 


\section{Introdução}

Em dezembro de 2019 sugiram, na China, mais especificamente na província Hubei e cidade de Wuhan, casos de pneumonia associada a síndrome respiratória aguda grave (SARS). A doença estava sendo causada por um novo coronavírus e foi nomeada pela Organização Mundial de Saúde (OMS) como COVID-19, sendo o vírus batizado cientificamente como SARS-CoV-2 (Severe Acute Respiratory Syndrome coronavirus 2). Devido à sua alta transmissibilidade, a COVID-19 que teve início na China espalhou-se rapidamente pela Europa e pelo mundo, de modo que em 11 de março de 2020 a OMS declarou estado de pandemia. (Adhikari et al., 2020; Saxena, 2020).

No Brasil o primeiro caso foi notificado no dia 26 de fevereiro de 2020. Os dados epidemiológicos mundiais e brasileiros em relação a essa doença são atualizados a cada dia. Segundo a página virtual Coronavirus Disease (COVID-19) Dashboard (https://covid19.who. int/) alimentada pela OMS, consulta realizada em agosto de 2020 mostra que mundo conta com aproximadamente 79,5 milhões de casos confirmados e cerca de 1,76 milhão de óbitos por COVID-19. No Brasil, por sua vez, são mais de 7,4 milhões de casos e mais de 190 mil mortes confirmados.

O termo quarentena diz respeito, conceitualmente, a um período de isolamento social para indivíduos que possivelmente tiveram exposição a um agente contagioso (14 dias no caso do SARS-CoV-2), porém está sendo popularmente utilizado para se referir a todo o período de distanciamento social e as regras em vigência durante pandemia pelo coronavírus, sendo este o significado adotado nesse artigo (WHO, 2020).Tanto na China, como em outros países, medidas para distanciamento social com restrições, ou mesmo proibições, de aglomerações foram adotadas a fim de prevenir o contágio entre as pessoas, como o fechamento de escolas, universidades, comércios, empresas e estabelecimentos de uso coletivo em geral (Dubey et al., 2020). Além disso, há recomendação, exigência e fiscalização massivas quanto aos cuidados pessoais, em especial, uso de máscara, higienização constante das mãos com água e sabão ou álcool em gel e distanciamento social, evitando aproximação e contato físico (Saxena, 2020; Organização Pan-americana de Saúde, 2020).

Com isso, muitos indivíduos tiveram alterações repentinas em suas rotinas e necessitaram se adaptar a uma nova realidade, na qual as famílias passaram a ficar mais tempo em suas casas, com novas dinâmicas de trabalho, estudo, atividades domésticas e cuidado com crianças e/ou idosos. Assim, o contexto da quarentena devido à COVID-19 torna-se um forte fator de risco para impactos negativos na saúde mental e qualidade de vida da população geral (Dubey et al., 2020; Holmes et al., 2020). O impacto econômico, o distanciamento físico, as privações das atividades extradomiciliares, o risco de contrair o vírus e o excesso de informações midiáticas são alguns fatores potenciais que vem gerando ou agravando sintomas como ansiedade, estresse, medo, raiva, culpa, angústia, insônia, sentimento de solidão e/ou de aprisionamento e depressão (Saxena, 2020). De forma mais grave, essas questões podem evoluir para a automutilação e o suicídio, valendo ressaltar que o suicídio teve um aumento de $30 \%$ na epidemia de síndrome respiratória aguda grave (SARS) que ocorreu na Ásia entre 2002 e 2003 (Holmes et al., 2020).

O primeiro estudo nacional chinês de larga escala sobre estresse psicológico na população geral do país foi feito entre janeiro e fevereiro de 2020 e evidenciou, com mais de 52 mil respostas válidas em questionário online, que aproximadamente 35\% dos entrevistados manifestaram sofrimento psicológico. Os maiores índices eram entre as mulheres, jovens de 18 a 30 anos (provavelmente pelo maior acesso à informação), indivíduos com mais de 60 anos (maior ameaça por serem grupo de risco), pessoas com nível educacional mais alto (possivelmente por possuírem mais informações sobre os riscos à saúde) e trabalhadores migrantes (principalmente sintomas de angústia, pois sabem que estão submetidos a uma maior exposição em transporte público, porém possuem medo de perder o emprego) (Qiu et al., 2020).

Outro estudo chinês de corte transversal e de base populacional, com 56.679 participantes, utilizou questionário online entre 28 de fevereiro e 11 de março de 2020 , evidenciando que $27,9 \%$ relataram sintomas de depressão, 31,6\% sintomas de ansiedade, $29,2 \%$ sintomas de insônia e $24,4 \%$ sintomas de estresse agudo (Shi et al., 2020). A prevalência desses sintomas durante a pandemia foi maior do que antes do surto, quando as taxas de depressão moderada a grave, ansiedade moderada a grave e insônia na China eram por volta de 6,0\%,5,3\% e 15,0\%, respectivamente, segundo os próprios autores. 
Além da China, foco de início da COVID-19, acredita-se que este fenômeno tenha ocorrido também nos outros países, em maior ou menor intensidade, mas, principalmente, naqueles que sofreram e estão sofrendo forte impacto. Fala-se em uma "pandemia de medo" diante à ameaça da doença, medo este que aumentaria os níveis de estresse e ansiedade em pessoas mentalmente saudáveis e intensificaria transtornos psiquiátricos preexistente. Inclusive, as implicações de saúde mental tendem a ser mais prevalentes e duradouras que a própria pandemia (Ornell et al., 2020).

Pessoas que contraíram COVID-19 e seus familiares, profissionais de saúde, bem como idosos, portadores de comorbidades, crianças e seus respectivos cuidadores são grupos que tendem a ser mais afetados psicologicamente e demandam atenção especial (Saxena, 2020). Durante a quarentena houve um aumento dos casos de violência doméstica e contra a mulher, afetando negativamente as mães e o desenvolvimento de seus filhos. Sintomas de depressão, principalmente nas mães, já são frequentes, mas podem se agravar por conta do fechamento de creches e de escolas, pelo distanciamento social reduzindo a rede de apoio, por crise na saúde pública e pela recessão econômica (Núcleo Ciência pela Infância, 2020).

Vale destacar que no Brasil as famílias de baixa renda tendem a sofrer mais pela falta da escola pública e da merenda escolar, bem como pela carência de itens de necessidades básicas, precariedade no sistema de saúde e desemprego. Essa realidade leva a um estresse constante no ambiente familiar que, além de maléfico por si só, pode agravar situações em que a criança já não era bem cuidada, já existiam dificuldades financeiras e/ou relações familiares conflituosas de violência e maus-tratos (Núcleo Ciência pela Infância, 2020).

Os pais se deparam com a situação de conviver a maior parte do tempo, em um mesmo ambiente, com a família em que, além das demandas emocionais da convivência diária, somam-se o trabalho em home office, o trabalho doméstico e os cuidados com os filhos. Estes cuidados incluem o acompanhamento das atividades da escola, que agora são em casa, via plataforma digital. Os pais de crianças e adolescentes com transtorno mental, se deparam também com a demanda de manter a criança em casa, com as terapias também por plataforma digital, ou sem as terapias. Isso pode ser particularmente exigente para pais de crianças e adolescentes portadores de transtornos mentais como o Transtorno do Espectro Autista (TEA).

Segundo a $5^{\text {a }}$ edição do Manual Diagnóstico e Estatístico de Transtornos Mentais (DSM-5), o Transtorno do Espectro Autista (TEA) está dentro do grupo de Transtornos do Neurodesenvolvimento, sendo determinado por início precoce de déficits persistentes na comunicação e na interação social em diferentes contextos, associados a padrões restritos e repetitivos de comportamento, interesses ou atividades (APA, 2014). Estas alterações causam prejuízo funcional significativo nas diversas áreas da vida do indivíduo e muitas vezes há, concomitantemente, comprometimento intelectual e/ou de linguagem, podendo, ainda, apresentar comorbidades psiquiátricas, genéticas ou clínicas em geral. A OMS estima que 1 em cada 160 crianças seja portadora de TEA, não havendo ainda estimativas populacionais no Brasil (OPAS, 2017). Trata-se, portanto, de um transtorno muito prevalente, majoritariamente diagnosticado na infância, e que traz limitações no desenvolvimento, o que torna indispensável o acompanhamento de especialistas e cuidados especiais por parte dos seus responsáveis.

Crianças com TEA exigem adaptações na dinâmica familiar em aspectos como educação formal, estimulação sensorial, comunicação, rotina e atenção aos detalhes do desenvolvimento geral (Gomes et al., 2015). Trata-se de um contexto que gera preocupações, inseguranças, quebra de expectativas, sobrecarga física e emocional (DePape \& Lindsay, 2015). Tudo começa com o diagnóstico, que costuma trazer sentimentos de apreensão nos pais, diante da sensação de perda de um filho idealizado ou de um filho saudável (Aguiar \& Pondé, 2020). Sintomas de ansiedade e depressão são prevalentes entre pais de crianças com TEA, principalmente aqueles com sintomas comportamentais mais proeminentes (Machado Junior et al., 2016). O comportamento, a comunicação insuficiente e o déficit cognitivo dos filhos mostraram-se ser os sintomas mais relacionados ao estresse parental (Gomes et al., 2015).

Além dos desafios trazidos pela tríade sintomática característica do autismo, sintomas associados como hiperatividade, agitação psicomotorora, distúrbio do sono, comportamento auto e hetero lesivo podem levar a estresse emocional e sintomas emocionais nos pais (Machado Junior et al., 2016). O isolamento social exigido durante a pandemia fez com que os cuidados terapêuticos a essas crianças e adolescentes fossem 
bruscamente interrompidos (Narzisi, 2020), o que pode gerar retrocesso em relação à evolução terapêutica conquistada. A pandemia traz, portanto, além das demandas habituais para os pais, a ausência das atividades externas que costumavam ajudar na adaptabilidade da criança e do adolescente (Narzisi, 2020; Cahapay, 2020).

Ainda não existem estudos de revisão de literatura que sintetizem as produções científicas atuais sobre a saúde mental e qualidade de vida de pais de pessoas com TEA. Buscando preencher essa lacuna, este estudo tem como objetivo revisar a literatura científica que aborda o impacto das mudanças geradas pela pandemia da COVID-19 sobre a saúde mental e a qualidade de vida de pais de pessoas portadoras de TEA, dialogando com os resultados desses estudos com estudos prévios sobre esse tema.

\section{Metodologia}

Trata-se de uma revisão narrativa de literatura, sobre a saúde mental e qualidade de vida de pais com filhos portadores com TEA durante a pandemia da COVID-19. Diante da escassez literária sobre o assunto, conduziu-se uma metodologia baseada no guia do Grupo de Métodos de Revisões Rápidas de Cochrane (Garritty et al.,2020).

A pesquisa literária ocorreu entre fevereiro e março de 2021 e foi realizada em duas etapas, a fim de selecionar gradativamente estudos de maior a menor impacto científico, com prioridade aos estudos revisados por pares. A saber:

Etapa 1: Coletar artigos na base de dados PubMed/ MEdline.

Etapa 2: Coletar artigos nas bases de dados Google scholar, Scielo (Scientific Electronic Library Online), Portal BVS (Biblioteca Virtual em Saúde) e Periódicos Capes (Coordenação de Aperfeiçoamento de Pessoal de Nível Superior).
Em ambas as etapas foram selecionados artigos publicados a partir de dezembro de 2019, marco temporal do início da doença. O estudo limitou-se a artigos em língua inglesa e/ou em língua portuguesa, em busca de agregar dados brasileiros pertinentes. Os termos de busca, descritores em saúde e correlatos utilizados de acordo com DeCS e MeSH foram: parents (pais) AND autism spectrum disorder (transtorno do espectro autista) OR autistic disorder (transtorno autístico) AND mental health (saúde mental) $A N D /$ $O R$ quality of life (qualidade de vida) AND covid19 OR quarantine (quarentena).

Os artigos foram revisados e incluídos se abordassem dados empíricos sobre a saúde mental e/ou qualidade de vida de pais de indivíduos com TEA durante a pandemia da COVID-19. Como o objetivo deste estudo é discutir o impacto da pandemia nessa população, foram excluídos estudos de intervenção. Nos estudos selecionados, buscamos as seguintes informações: país de origem, número de participantes, características da amostra, período da coleta de dados, desenho do estudo, instrumentos de avaliação e resultados. Os resultados desses estudos foram analisados e discutidos comparando com estudos feitos antes do período da pandemia.

\section{Resultados}

Na etapa 1, foram identificados 219 artigos na base de dados PubMed/Medline, dos quais 209 foram excluídos por não abordarem saúde mental e/ou qualidade de vida, por não utilizarem a população desejada ou analisarem intervenção sobre a amostra. Um total de dez artigos foram selecionados. Na etapa 2, foram identificados 84 artigos, dos quais, 2 atenderam aos critérios de inclusão. Ao final, foram selecionados 13 artigos, todos de estudo qualitativos observacionais, dos quais cinco envolvem pais de indivíduos portadores de necessidades especiais, incluindo pais de portadores de TEA, e oito abordam especificamente esta população. A Tabela 1 resume a caracterização sintetizada de cada estudo. 
As medidas restritivas por conta da COVID-19 potencialmente afetam os pais e os seus filhos portadores de TEA, pois atrapalham ou impossibilitam atividades extradomiciliares como o acompanhamento terapêutico, escolas, creches e ambientes de lazer. Estudo americano demonstrou que $75 \%$ dos pais relataram nível de estresse moderado ou severo devido à interrupção dos serviços de apoio e terapias de seus filhos, principalmente nas idades pré-escolar e escolar (White et al., 2020). Durante a quarenta, os pais de crianças e adolescentes com TEA têm apresentado os seguintes problemas relacionados ao impacto que estão sofrendo na saúde mental e bem-estar: (1) mudança ou perda do emprego e insegurança/instabilidade financeira; (2) surgimento ou aumento de sintomas de estresse e ansiedade; (3) relações conflituosas familiares, incluindo entre pais e filhos; (4) excesso de responsabilidades (Albaum et al., 2020).

Duas pesquisas com pais de crianças portadoras de TEA foram realizadas na Arábia Saudita durante a pandemia no ano de 2020. O primeiro estudo publicado realizou uma pesquisa online com 150 participantes e não levou em consideração possíveis comorbidades presentes nos filhos. Este estudo demonstrou que a maioria dos pais relataram que seu nível de estresse aumentou e seu bem-estar emocional foi afetado durante o contexto da COVID-19. Os aspectos que afetaram negativamente a saúde mental parental foram: a idade menor do filho (a), menor frequência e utilidade de suporte ao filho com TEA (sendo que foi considerado suporte ao TEA na pandemia qualquer apoio recebido da escola, serviços locais de saúde e/ou terapeutas) e maior severidade de sintomas e comportamentos autistas (Alhuzimi, 2021). O segundo estudo, por sua vez, não incluiu pais de crianças com outra comorbidade além do TEA, totalizando 211 participantes sauditas. Este evidenciou aumento estatisticamente significante do estado de ansiedade parental entre antes e durante a pandemia. Maior nível de ansiedade nos pais relacionou-se a pais mais jovens (26 a 35 anos de idade), pais do sexo feminino (mães), filhos na faixa etária de 8 a 12 anos de idade e filhos do sexo feminino. Quando questionados sobre suas necessidades em relação ao cuidado da saúde mental, os pais relataram maior procura por apoio psicológico, principalmente para mães, tal como apoio financeiro, sessões e aconselhamentos online e rede de apoio com troca de experiências.
Há evidências de que o contexto da pandemia tem provocado piora significativa quanto a qualidade do sono, a hipersensibilidade e as alterações de comportamento de indivíduos portadores de TEA, como aumento de irritabilidade e hiperatividade (Colizzi et al., 2020; Mutluer et al., 2020, Türkoğlu et al., 2020). Estudo realizado na Turquia fez uso de questionário online em que os pais responderam principalmente sobre o impacto da pandemia sobre seus filhos com uso de perguntas originais e das escalas $A B C$ (Aberrant Behavior Checklist) e PSQI (Pittsburgh Sleep Quality Index). Entretanto, também foi utilizado o BAI (Back Anxiety Inventory) para avaliar o nível de ansiedade dos cuidadores primários dos participantes, demonstrando que essas mudanças estão diretamente relacionadas a maiores níveis de ansiedade neles. $46 \%$ dos pais relataram sintomas de ansiedade moderados a severos e a ansiedade parental teve correlação significativa com o escore total dos filhos na escala ABC durante a pandemia (Mutluer et al., 2020).

O único estudo encontrado que avaliou especificamente qualidade de vida foi uma pesquisa realizada no Japão com pais de crianças com necessidades especiais, sendo $65 \%$ delas portadoras de TEA. A média de qualidade de vida dos pais foi 63,8 de um total de 100 pontos na escala WHOQOL-bref. Crianças com piora no padrão de sono e mães com padrões habituais de trabalho foram fatores que reduziram a qualidade de vida da família, com aumento do estresse parental, níveis sérios de ansiedade e depressão, e sintomas graves internalizantes e externalizantes em crianças (Ueda et al., 2020). Foram encontrados três estudos chineses, dos quais um analisou especificamente pais de portadores de TEA e dois analisaram pais de crianças com necessidades especiais, incluindo pais de autistas. O estudo que usou população específica dividiu sua amostra em dois grupos: grupo de pais de portadores de TEA com 1.764 participantes e grupo de pais de indivíduo com desenvolvimento típico (DT) com 4.962 participantes. Na análise da saúde mental dessas pessoas durante a pandemia da COVID-19, observou-se que os escores de ansiedade e depressão foram significativamente maiores no grupo que tinha filhos com TEA. Não obstante, as mães de indivíduos autistas apresentaram maiores níveis de sintomas de ansiedade e depressão do que os pais e do que os cuidadores de crianças com DT, o que apresentou e associação (Wang et al., 2021). 
Outro estudo que utilizou grupo controle foi realizado em Portugal com 99 participantes, sendo 43 pais de filhos com TEA e 56 pais de crianças com DT. Neste, foi observado que pais de autistas relatam maiores mudanças de comportamento de seus filhos e maior dificuldade de gerenciamento emocional. Os pais relataram maiores níveis de ansiedade em si do que em seus filhos e os pais de portadores de TEA mostraram-se mais ansiosos (Amorim et al., 2020). Chen (2020) analisou a saúde mental na população de pais de indivíduos com TEA (31,3\%), com deficiência intelectual $(48,5 \%)$ ou deficiência visual/dificuldade auditiva (20,2\%). Seus resultados mostraram que pais de indivíduos com TEA são mais susceptíveis a problemas na saúde mental quando comparados com os outros transtornos, o que mostrou estar relacionado a alterações de comportamento da criança, relação disfuncional entre pai e filho, bem como demandas psicológicas parentais e falta de suporte de amigos, familiares e profissionais (Chen et al., 2020). Em estudo semelhante, Ren (2020) utilizou amostra com $31,2 \%$ de pais de autistas, $48,2 \%$ pais de deficientes intelectuais e $20,6 \%$ de pais de crianças com deficiências visual ou auditiva. Diferente de estudos aqui citados, este não encontrou relação entre estado de ansiedade parental e gênero ou idade da criança. Maiores níveis de ansiedade estiveram relacionados a menor nível educacional e socioeconômico. Embora o escore de ansiedade tenha sido bastante superior em pais de crianças com TEA, não houve diferença estatisticamente significante entre os grupos. Por fim, demonstra-se que menos apoio social combinado com maiores níveis de problemas mentais e comportamentais gera mais estresse parental na pandemia (Ren et al., 2020).

Asbury (2020) avaliou 241 pais de crianças com deficiências e necessidades educacionais especiais no Reino Unido solicitando-os que descrevessem livremente suas percepções sobre o impacto da COVID-19 na sua saúde mental e na de seu filho. $82 \%$ da amostra eram pais de crianças com TEA, $32 \%$ com dificuldades sociais, emocionais e de saúde mental, 23\% com Transtorno de Déficit Atenção e Hiperatividade, $19 \%$ com transtorno do desenvolvimento da linguagem, dentro outros transtornos e deficiências físicas. Os participantes relatam que a pandemia levou ao aumento de ansiedade, medo, angústia, mau humor e estresse. Novamente, os dados apontam que mais pais do que crianças experimentaram aumento de ansiedade (44\% vs $25 \%)$ e estresse (12\% vs $5 \%$ ). Alguns pais relataram se sentir oprimidos e descreveram o impacto da dificuldade de compreensão e entendimento da criança sobre o contexto (Asbury et al., 2020). Na Índia, outra pesquisa com cuidadores de crianças com necessidades especiais foi realizada, sendo que $25 \%$ cuidavam de autistas. As prevalências de sintomas de depressão, ansiedade e estresse foram, respectivamente, $62,5 \%, 20,5 \%$ e $36,4 \%$, e o esforço do cuidador no cuidado à criança teve um aumento estatisticamente significante de antes para durante a pandemia. Evidenciou-se relação relevante entre sintomas de depressão, ansiedade e estresse com o nível de esforço do cuidador (Dhiman et al., 2020).

Por fim, o estudo com menor rigor científico foi feito na Sérvia durante a pandemia e a política de lockdown no país e contou com 85 participantes, pais de crianças com TEA, que completaram virtualmente questionário validado modificado para que os problemas fossem diretamente ligados à emergente pandemia (Stankovic et al., 2020). Os resultados mostram que $60 \%$ refere sentimento de desemparo, $87 \%$ necessita de ferramentas adicionais de educação para os filhos durante a quarentena e $58 \%$ se encontra totalmente insatisfeito com o apoio recebido dos serviços de saúde e educação. Os pais levantaram desafios como problema em proporcionar assistência social adequada às crianças, falta do ensino formal, dificuldade de conseguir descansar, acesso a máscara e luvas de proteção e garantia dos direitos humanos fundamentais (Stankovic et al., 2020). Os resultados mostram que: $84,4 \%$ das crianças não receberam ajuda em casa ou educação adicional relevante; em $15,3 \%$ os professores enviavam planos semanais de aprendizado ou os filhos eram ensinados por um profissional; o sentimento de desamparo durante a pandemia e emergência é identificado por 59,8\%; 58,4\% estão totalmente insatisfeitos com o apoio que recebem em casa; $74,1 \%$ dos pais afirmaram que é importante o contato com os serviços. Os pais relataram que vem enfrentando os seguintes desafios: proporcionar condições sociais adequadas de assistência / apoio à criança $(51,2 \%)$, falta de educação escolar $(48,8 \%)$, não conseguir descansar $(41,3 \%)$, fornecimento de máscaras e luvas de proteção $(40 \%)$, proteção dos direitos humanos fundamentais (35\%). O estudo é um rascunho ainda não revisado e que não apresentou gráficos de seus resultados (Stankovic et al., 2020). 
Tabela 1. Caracterização dos estudos selecionados

\begin{tabular}{|c|c|c|c|c|c|}
\hline Autores & País & $\begin{array}{l}\text { População do } \\
\text { estudo }\end{array}$ & $\begin{array}{l}\mathrm{N} \text { da } \\
\text { amostra }\end{array}$ & $\begin{array}{l}\text { Instrumentos de } \\
\text { avaliação }\end{array}$ & Resultados principais \\
\hline ALTHIABI et al. & $\begin{array}{l}\text { Arábia } \\
\text { Saudita }\end{array}$ & $\begin{array}{l}\text { Pais de crianças } \\
\text { com TEA e sem } \\
\text { comorbidades }\end{array}$ & 211 & $\begin{array}{l}\text { Questionário online com } \\
\text { perguntas originais e as } \\
\text { escalas HADS (Hospital } \\
\text { Anxiety and Depression } \\
\text { Scale) e GHQ-12 (General } \\
\text { Health Questionnaire) }\end{array}$ & $\begin{array}{l}\text { Comparado com antes } \\
\text { da pandemia, os pais } \\
\text { estão mais ansiosos } \\
\text { O nível de ansiedade } \\
\text { relacionou-se ao número } \\
\text { de crianças com TEA. } \\
\text { Pais mais novos e pais de } \\
\text { meninas mostraram-se } \\
\text { mais ansiosos. } \\
\text { Ansiedade durante a } \\
\text { pandemia piorou o } \\
\text { estado de saúde mental } \\
\text { dos pais. }\end{array}$ \\
\hline ALHUZIMI et al. & $\begin{array}{l}\text { Arábia } \\
\text { Saudita }\end{array}$ & $\begin{array}{l}\text { Pais de crianças } \\
\text { com TEA }\end{array}$ & 150 & $\begin{array}{l}\text { Questionário online com } \\
\text { perguntas originais e as } \\
\text { escalas PSI-SF (Parent } \\
\text { Stress Index Short Form) } \\
\text { e GHQ-12 (General } \\
\text { Health Questionnaire) }\end{array}$ & $\begin{array}{l}\text { Estresse e/ou sofrimento } \\
\text { e/ou bem-estar } \\
\text { emocional dos pais } \\
\text { foram impactados por: } \\
\text { idade da criança (maior } \\
\text { impacto com filhos mais } \\
\text { novos), severidade dos } \\
\text { sintomas e } \\
\text { comportamentos de TEA } \\
\text { e frequência e utilidade } \\
\text { de meios de suporte ao } \\
\text { TEA. } \\
\text { O bem-estar emocional } \\
\text { impactou o nível de } \\
\text { estresse parental. }\end{array}$ \\
\hline MUTLUER et al. & Turquia & $\begin{array}{l}\text { Pacientes com } \\
\text { TEA e sem } \\
\text { doença } \\
\text { neurológica } \\
\text { grave ou } \\
\text { síndrome } \\
\text { genética } \\
\text { complexa. } \\
\text { Pesquisa } \\
\text { respondida } \\
\text { pelos pais. }\end{array}$ & 87 & $\begin{array}{l}\text { Questionário online com } \\
\text { perguntas originais e } \\
\text { escalas voltadas aos } \\
\text { portadores de TEA. BAI } \\
\text { (Back Anxiety Inventory) } \\
\text { foi utilizado para } \\
\text { mensurar o nível de } \\
\text { ansiedade dos } \\
\text { cuidadores primários dos } \\
\text { participantes. }\end{array}$ & $\begin{array}{l}25 \% \text { dos cuidadores } \\
\text { primários de indivíduos } \\
\text { com TEA apresentaram } \\
\text { nível mínimo de } \\
\text { ansiedade, } 29 \% \text { nível } \\
\text { médio, } 21 \% \text { nível } \\
\text { moderado e } 25 \% \text { nível } \\
\text { severo. } \\
\text { Pior nível de ansiedade } \\
\text { parental diante pior } \\
\text { escore total dos filhos na } \\
\text { escala ABC durante a } \\
\text { pandemia. }\end{array}$ \\
\hline WANG et al. & China & $\begin{array}{l}\text { Pais de crianças } \\
\text { com TEA e pais } \\
\text { de crianças } \\
\text { com } \\
\text { desenvolviment } \\
\text { o típico. }\end{array}$ & 6.726 & $\begin{array}{l}\text { Questionário online com } \\
\text { perguntas originais e } \\
\text { escalas: CD-RISC } \\
\text { (Connor-Davidson } \\
\text { Resilience Scale) SCSQ } \\
\text { (Simplified Coping Style } \\
\text { Questionnaire), SAS } \\
\text { (Selfrating Anxiety Scale) } \\
\text { e SDS (Self-rating } \\
\text { Depression Scale). }\end{array}$ & $\begin{array}{l}\text { Maiores índices de } \\
\text { ansiedade e depressão } \\
\text { em pais de filhos com } \\
\text { TEA comparados a pais } \\
\text { de filhos com DT. } \\
\text { Ter filho com TEA } \\
\text { apresentou associação } \\
\text { com piores índices de } \\
\text { ansiedade e depressão } \\
\text { em mães na pandemia. }\end{array}$ \\
\hline
\end{tabular}


Tabela 1. Caracterização dos estudos selecionados

\begin{tabular}{|c|c|c|c|c|c|}
\hline Autores & País & $\begin{array}{l}\text { População do } \\
\text { estudo }\end{array}$ & $\begin{array}{l}\mathrm{N} \mathrm{da} \\
\text { amostra }\end{array}$ & $\begin{array}{l}\text { Instrumentos de } \\
\text { avaliação }\end{array}$ & Resultados principais \\
\hline WHITE et al. & EUA & $\begin{array}{l}\text { Pais ou } \\
\text { cuidadores } \\
\text { primários de } \\
\text { crianças com } \\
\text { TEA }\end{array}$ & 3.502 & $\begin{array}{l}\text { Questionário online com } \\
\text { perguntas originais } \\
\text { relacionadas ao impacto } \\
\text { da interrupção do } \\
\text { cuidado ao filho autista e } \\
\text { escala BFDS (Brief Family } \\
\text { Distress Scale). }\end{array}$ & $\begin{array}{l}75 \% \text { dos pais relataram } \\
\text { nível de estresse } \\
\text { moderado ou severo } \\
\text { devido à interrupção dos } \\
\text { serviços de apoio e } \\
\text { terapias de seus filhos, } \\
\text { principalmente nas } \\
\text { idades pré-escolar e } \\
\text { escolar. }\end{array}$ \\
\hline AMORIM et al. & Portugal & $\begin{array}{l}\text { Crianças com } \\
\text { TEA e crianças } \\
\text { com DT e seus } \\
\text { pais }\end{array}$ & 99 & $\begin{array}{l}\text { Questionário online com } \\
\text { perguntas originais, } \\
\text { sendo que a nível de } \\
\text { ansiedade e adaptação à } \\
\text { quarentena foram } \\
\text { referidas numa escala de } \\
0 \text { a } 10 .\end{array}$ & $\begin{array}{l}\text { Pais de autistas relatam } \\
\text { maiores mudanças de } \\
\text { comportamento de seus } \\
\text { filhos e maior dificuldade } \\
\text { de gerenciamento } \\
\text { emocional. } \\
\text { Pais relataram maiores } \\
\text { níveis de ansiedade em s } \\
\text { do que em seus filhos e } \\
\text { os pais de portadores de } \\
\text { TEA estão mais ansiosos } \\
\text { na pandemia. }\end{array}$ \\
\hline STANKOVIC et al. & Sérvia & $\begin{array}{l}\text { Pais ou } \\
\text { cuidadores } \\
\text { primários de } \\
\text { crianças com } \\
\text { TEA }\end{array}$ & 85 & $\begin{array}{l}\text { Questionário online } \\
\text { baseado em Caregiver } \\
\text { Needs Survey, com } \\
\text { modificações para } \\
\text { direcionar as questões à } \\
\text { situação de pandemia e } \\
\text { estado de emergência. }\end{array}$ & $\begin{array}{l}60 \% \text { referiu sentimento } \\
\text { de desemparo, } 87 \% \\
\text { necessita de ferramentas } \\
\text { adicionais de educação } \\
\text { para os filhos durante a } \\
\text { quarentena e } 58 \% \text { se } \\
\text { encontra totalmente } \\
\text { insatisfeito com o apoio } \\
\text { recebido dos serviços de } \\
\text { saúde e educação. }\end{array}$ \\
\hline DHIMAN et al. & Índia & $\begin{array}{l}\text { Cuidadores de } \\
\text { crianças com } \\
\text { necessidades } \\
\text { especiais }\end{array}$ & 264 & $\begin{array}{l}\text { Questionário online com } \\
\text { perguntas originais e } \\
\text { escala DASS-21 } \\
\text { (Depression Anxiety } \\
\text { Stress-21 Scale) e CSI } \\
\text { (Caregiver Strain Index). }\end{array}$ & $\begin{array}{l}\text { As prevalências sintomas } \\
\text { de depressão, ansiedade } \\
\text { e estresse foram, } \\
\text { respectivamente, } 62,5 \% \text {, } \\
20,5 \% \text { e } 36,4 \% \text {. } \\
\text { O esforço do cuidador no } \\
\text { cuidado à criança teve } \\
\text { um aumento } \\
\text { estatisticamente } \\
\text { significante de antes } \\
\text { para durante a } \\
\text { pandemia. } \\
\text { Evidenciou-se relação } \\
\text { entre sintomas de } \\
\text { depressão, ansiedade e } \\
\text { estresse com o nível de } \\
\text { esforço do cuidador. }\end{array}$ \\
\hline CHEN et al. & China & $\begin{array}{l}\text { Pais de crianças } \\
\text { com } \\
\text { necessidades } \\
\text { especiais }\end{array}$ & 1.450 & $\begin{array}{l}\text { Questionário online com } \\
\text { perguntas originais, } \\
\text { incluindo questões sobre } \\
\text { a demanda psicológica } \\
\text { dos pais durante a } \\
\text { COVID-19. E as escalas: } \\
\text { GHQ-12 (General Health } \\
\text { Questionnaire), PSS } \\
\text { (Perceived Social } \\
\text { Support), PSI-SF } \\
\text { (Parenting Stress Index- } \\
\text { Short Form) e NEO-FFI } \\
\text { (Neuroticism } \\
\text { Extraversion Openness } \\
\text { Five Factor Inventory) }\end{array}$ & $\begin{array}{l}\text { Pais de crianças com TEA } \\
\text { apresentam pior saúde } \\
\text { mental na pandemia } \\
\text { quando comparado a } \\
\text { pais de crianças com } \\
\text { outras necessidades } \\
\text { especiais. }\end{array}$ \\
\hline
\end{tabular}


Tabela 1. Caracterização dos estudos selecionados

\begin{tabular}{|c|c|c|c|c|c|}
\hline Autores & País & $\begin{array}{l}\text { População do } \\
\text { estudo }\end{array}$ & $\begin{array}{l}\mathrm{N} \text { da } \\
\text { amostra }\end{array}$ & $\begin{array}{l}\text { Instrumentos de } \\
\text { avaliação }\end{array}$ & Resultados principais \\
\hline REN et al. & China & $\begin{array}{l}\text { Pais de crianças } \\
\text { com } \\
\text { necessidades } \\
\text { especiais }\end{array}$ & 1.451 & $\begin{array}{l}\text { Questionário online com } \\
\text { perguntas originais sobre } \\
\text { problemas mentais e de } \\
\text { comportamento nas } \\
\text { crianças e nos pais } \\
\text { durante a COVID-19 e } \\
\text { escalas: PSI-SF } \\
\text { (Parenting Stress Index- } \\
\text { Short Form), } \\
\text { MSPSS(Multidimensional } \\
\text { Scale of Perceived Social } \\
\text { Support), NEO-FFI } \\
\text { (Neuroticism } \\
\text { Extraversion Openness } \\
\text { Five Factor Inventory) e } \\
\text { State Anxiety Inventory } \\
\text { (S-AI) in the State-Trait } \\
\text { Anxiety } \\
\text { Inventory (STAI). }\end{array}$ & $\begin{array}{l}\text { Embora o escore de } \\
\text { ansiedade tenha sido } \\
\text { bastante superior em } \\
\text { pais de crianças com TEA, } \\
\text { não houve diferença } \\
\text { estatisticamente } \\
\text { significante entre os } \\
\text { grupos. } \\
\text { Menos apoio social } \\
\text { combinado com maiores } \\
\text { níveis de problemas } \\
\text { mentais e } \\
\text { comportamentais gera } \\
\text { mais estresse parental } \\
\text { na pandemia. }\end{array}$ \\
\hline ASBURY et al. & $\begin{array}{l}\text { Reino } \\
\text { Unido }\end{array}$ & $\begin{array}{l}\text { Pais de crianças } \\
\text { com } \\
\text { deficiências e } \\
\text { necessidades } \\
\text { educacionais } \\
\text { especiais }\end{array}$ & 241 & $\begin{array}{l}\text { Os participantes } \\
\text { responderam de forma } \\
\text { livre à questão: } \\
\text { "Por favor descreva em } \\
\text { suas palavras como o } \\
\text { surto do coronavírus está } \\
\text { afetando sua saúde } \\
\text { mental e a saúde mental } \\
\text { dos seus filhos." }\end{array}$ & $\begin{array}{l}\text { Os participantes relatam } \\
\text { que a pandemia levou ao } \\
\text { aumento de ansiedade, } \\
\text { medo, angústia, mau } \\
\text { humor e estresse. Os } \\
\text { dados sugerem que mais } \\
\text { pais do que crianças } \\
\text { experimentaram } \\
\text { aumento de ansiedade } \\
\text { ( } 44 \% \text { vs } 25 \% \text { ) e estresse } \\
\text { ( } 12 \% \text { vs } 5 \%) \text {. } \\
\text { Alguns pais relataram se } \\
\text { sentir oprimidos e } \\
\text { descreveram o impacto } \\
\text { da dificuldade de } \\
\text { compreensão e } \\
\text { entendimento da criança } \\
\text { sobre o contexto }\end{array}$ \\
\hline UEDA et al. & Japão & $\begin{array}{l}\text { Crianças com } \\
\text { transtorno no } \\
\text { neurodesenvolv } \\
\text { imento e seus } \\
\text { pais }\end{array}$ & 136 & $\begin{array}{l}\text { Questionário online com } \\
\text { perguntas originais e, } \\
\text { para os pais, foram } \\
\text { utilizadas as escalas } \\
\text { WHO-Qol-bref, CES-D } \\
\text { (Center for Epidemiologic } \\
\text { Studies Depression } \\
\text { Scale), STAl (State-trait } \\
\text { Anxiety Inventory) e PSI } \\
\text { (Parenting Stress Index). }\end{array}$ & $\begin{array}{l}\text { A média de qualidade de } \\
\text { vida dos pais foi } 63,8 \text { de } \\
\text { um total de } 100 \text { pontos } \\
\text { na escala WHOQOL-bref. } \\
\text { Crianças com piora no } \\
\text { padrão de sono e mães } \\
\text { com padrões habituais } \\
\text { de trabalho foram } \\
\text { fatores que reduziram a } \\
\text { qualidade de vida da } \\
\text { família, assim como } \\
\text { aumento do estresse } \\
\text { parental, níveis sérios de } \\
\text { ansiedade e depressão e } \\
\text { sintomas graves de } \\
\text { internalização e } \\
\text { externalização em } \\
\text { crianças. }\end{array}$ \\
\hline
\end{tabular}




\section{Discussão}

A pandemia gerada pelo novo coronavírus é uma realidade que vem afetando direta ou indiretamente todos os setores da sociedade, forçando uma reformulação do convívio e das atividades. O distanciamento social da quarentena e a ameaça da COVID-19 vêm prejudicando a saúde mental e a qualidade de vida da população em geral, notadamente de grupos mais vulneráveis, como profissionais de saúde, idosos, crianças e/ou indivíduos com comorbidades clínicas ou psiquiátricas e os seus familiares.

Este estudo teve como objetivo revisar a literatura científica sobre a saúde mental de pais de crianças e adolescentes com TEA. A razão para essa preocupação é evidenciada por um corpo de estudos que indica a vulnerabilidade de pais de pessoas com TEA ao sofrimento psíquico, seja pelos desafios inerentes a cuidar de uma criança com tantas demandas (Machado Junior et al., 2016; Dey et al., 2019; Vasilopoulou \& Nisbet, 2016); seja pela vulnerabilidade genética desses pais para transtornos mentais (Xie et al., 2019).

\section{Saúde mental e qualidade de vida de pais de por- tadores de TEA}

Um estudo de metanálise, que avaliou a qualidade de vida (QV) de pais de crianças com doença mental, mostrou que os pais de filhos com TEA têm índices menores de qualidade de vida do que pais de crianças com enurese noturna e esquizofrenia (Dey et al., 2019). Outra revisão sistemática indicou uma pior QV entre pais de crianças com TEA em comparação com pais de crianças com desenvolvimento típico, envolvendo aspectos relacionados às dificuldades comportamentais da criança, desemprego dos pais, ser mãe e a falta de apoio social (Vasilopoulou \& Nisbet, 2016).

Fatores importantes que afetam a qualidade de vida dos pais dessas crianças são as necessidades financeiras maiores e as dificuldades no acesso a serviços de saúde e apoio social. Em relação ao financeiro, sabe-se que os pais de portadores de TEA tendem a ter maiores jornadas de trabalho para arcar com terapia, educação voltada aos autistas e acompanhamento médico (Gomes et al., 2015). Muitas mães acabam recebendo salários menores ou abdicando de trabaIhar para cuidar dos filhos autistas, o que diminui a renda familiar. Ademais, os serviços de saúde, educação e lazer costumam ser insuficientes por si só, falta suporte para a inclusão desses indivíduos e ainda há uma grande estigmatização (OPAS, 2017). Isso tende a piorar na nova realidade da quarentena, tanto por conta do distanciamento social, que suspendeu muitos atendimentos presenciais públicos e privados, atividades escolares e espaços de convivência, como também pelo impacto econômico negativo significativo para as famílias.

Os pais que não costumam ser os protagonistas nos cuidados da criança passaram a assumir na quarentena o papel de cuidador principal ou único cuidador de seus filhos. Nos EUA, cerca de $24 \%$ das crianças que vivem em casas monoparentais estão sob os cuidados de um pai (i.e., genitor masculino) solteiro (Frye, 2016). Um estudo americano com dez pais de crianças com TEA indicou que as necessidades deles envolviam conquistar dinheiro suficiente para arcar com as terapias, consultas e tratamentos; trabalhar em equipe com a família, professores, terapeutas e amigos; encontrar profissionais de saúde honestos e que passassem informações corretas; buscar ter mais envolvimento e mais tempo em relação aos cuidados com o(s) filho(s) e com a família (Frye, 2016).

Uma pesquisa britânica utilizou questionários online com 109 familiares de adultos autistas e mostrou que dois terços obtiveram escores indicativos de depressão leve ou moderada, três quartos referiram ansiedade e preocupação e metade autorrelataram níveis clínicos de estresse (Herrema et al., 2017). Nesta pesquisa foram utilizadas escalas de intolerância para incerteza (Intolerance of Uncertainty Scale, IUS), incluindo uma voltado para os pais. Tal questionário tem como objetivo avaliar o impacto psicológico diante de situação incertas, ambíguas ou imprevisíveis, o que pode ser aplicável à situação atual de pandemia. A intolerância à incerteza no adulto autista e no seu cuidador contribui para que este fique mais preocupado, estressado e ansioso (Herrema et al., 2017).

Questões relacionadas ao TEA que preocupam os pais são: precisar lidar com maior quantidade de tarefas, adaptações ao convívio social, superação das dificuldades e da estigmatização, falta de apoio social, bem como buscar oferecer o suficiente financeiramente para melhor desenvolvimento do filho e, consequentemente, a conquista do melhor futuro possível para ele (DePape \& Lindsay, 2015). Muitas vezes, os pais negligenciam o autocuidado, abalando a sua saúde mental e qualidade de vida. Apesar de ser inegável a necessidade de suporte psicossocial para os pais de 
indivíduos com TEA, o acesso a esse suporte ainda não é facilitado e abrangente, e o cenário pandêmico pode se constituir em mais um obstáculo.

\section{Saúde mental e qualidade de vida de pais de portadores de TEA na pandemia COVID-19}

Indivíduos com TEA podem ter dificuldade de compreender o que é a COVID-19 e as mudanças ambientais que a doença acarreta, o que pode dificultar a sua adaptação às medidas de distanciamento social, como ficar em casa por muito tempo e cumprir as orientações de higiene. Características de alguns indivíduos autistas, como a hipersensibilidade sensorial, podem dificultar o uso da máscara e impedir uma proteção adequada (Mutluer et al., 2020). Todos esses obstáculos criam um ambiente de risco para a saúde mental e qualidade de vida de seus cuidadores. Por conta de tantas instabilidades, particularidades e adequações no cenário da pandemia, os pais de crianças e adolescentes com TEA tendem a apresentar maior sofrimento mental e, portanto, necessitam de suporte psicossocial específico.

A pesquisa pioneira que avaliou o impacto da pandemia no bem-estar de pais e crianças (entre 2 e 14 anos de idade) é italiana e contou com a participação de 854 pais que responderam a questionários online. 0 estudo mostrou que o estresse parental e os problemas psicológicos dos filhos estavam associados à percepção que os pais tinham sobre a pandemia e o quanto desafiador estava sendo para eles lidar com as adaptações exigidas durante o período avaliado (Spinelli et al., 2020). Para famílias com membros com TEA essa realidade pode ser ainda mais séria. A literatura mostra que em outros surtos de doença transmissíveis como H1N1 e SARS, um terço das crianças e um quarto dos pais que foram submetidos a isolamento social ou quarentena manifestaram Transtorno do Estresse Pós-Traumático (TEPT) (Sprang et al., 2013; Mutluer et al., 2020).

Colizzi (2020) realizou um estudo, também na Itália, que analisou o impacto psicossocial e comportamental da COVID-19 em pessoas com TEA. Este estudo também utilizou questionário online, composto por 38 questões de múltipla escolha e duas questões abertas referentes a: características sociodemográficas e clínicas de indivíduos com TEA, impacto do surto de COVID-19 em seu bem-estar, e necessidade de lidar com a emergência. Participaram do estudo 527 pais e responsáveis de indivíduos com TEA.
Os resultados indicaram que eles possuem dificuldade em gerenciar as tarefas diárias, lidar com os problemas de comportamento das crianças, e que estão precisando de assistência médica e ajuda com os serviços domésticos (Colizzi et al., 2020). O estudo foi feito com pais de pessoas com TEA, porém não abordou a saúde mental deles, mas, sim, a dos filhos. A emergência COVID-19 resultou em um período desafiador para $93,9 \%$ das famílias, aumentando as dificuldades no gerenciamento das atividades diárias; $35,5 \%$ das crianças apresentando problemas de comportamento mais intensos e $41,5 \%$ mais recorrentes; problemas de comportamento pré-existentes à pandemia previu um maior risco de intensificarem e de se tornarem mais frequentes; as necessidades relatadas incluíam: receber mais suporte de saúde $(47,4 \%)$, especialmente suporte em casa $(29,9 \%)$ e intervenções para combater uma quarentena potencialmente perturbadora $(16,8 \%)$.

O estudo feito na Sérvia que abordou diretamente a saúde mental dos pais indica que a maioria refere sentimento de desamparo e necessidade de ferramentas adicionais de educação para os filhos na pandemia; ao tempo em que estão totalmente insatisfeitos com o apoio recebido dos serviços de saúde e educação (Stankovic et al., 2020). Apesar de ser um rascunho, a pesquisa mostra a situação de desamparo desses pais, diante de necessidade de isolamento social com filhos que demandam cuidados tão especializados.

Apesar da ausência de estudos empíricos, serviços e profissionais envolvidos com a clínica de crianças tem feito sugestões para um melhor convívio familiar para pais e crianças, tais como: respeitar rotinas, estabelecer e manter horários, limitar uso de eletrônicos, preservar horários lúdicos, organizar e separar um espaço para cada atividade, manter atividades físicas durante o dia e atividades mais tranquilas à noite, evitar excesso de notícias negativas, compreender que as crianças podem ter alterações funcionais ou comportamentais que demandam paciência e tolerância, buscar fontes de informações úteis e positivas para o dia-a-dia, manter rotina pessoal e autocuidado, dividir tarefas e exercer a autonomia de cada membro familiar dentro das possibilidades (Núcleo Ciência pela Infância, 2020).

O psicólogo e pesquisador italiano Antonio Narzisi lançou um editorial com dez dicas para ajudar pais e cuidadores crianças portadoras de TEA neste período de quarentena. Ele sugere que os pais e os cuidadores contem com a ajuda de especialistas para construir e 
manter uma rotina funcional com os seus filhos em casa. O autor sugere: (1) explicar de forma simples e concreta, podendo usar a estratégia de comunicação alternativa, o que é a COVID-19 e o porquê é necessário permanecer em casa no momento; (2) planejar as atividades diárias, com espaço e momento próprios para cada uma, o que pode ajudar a organizar o pensamento e a desenvolver interação com a família; (3) utilizar atividades lúdicas semiestruturadas que facilitem o despertar de interesse e o envolvimento da criança; (4) usar também jogos sérios, com foco educacional, que estimulem o reconhecimento de expressões faciais, gestos, sentimentos e situações; (5) fazer um uso compartilhado do videogame, ou outro conteúdo virtual de consumo da criança, com os pais e familiares, evitando o seu isolamento e longas permanências acessando a internet sozinha; (6) valorizar e planejar atividades para compartilhar com os pais os interesses especiais que a criança possa ter; (7) psicoterapia online, principalmente para crianças com TEA de alto funcionamento e que já faziam sessões presenciais anteriormente; (8) consultas semanais online de psicoterapia para pais e cuidadores, que podem ajudar no controle de estresse e ansiedade gerados ao ficarem sozinhos em casa cuidando dos filhos; (9) manter o contato com professores e colegas da escola estimulando a interação perdida sem o convívio social físico, pode ser por meio de vídeochamadas, ligações telefônicas ou cartas; (10) manter um tempo livre sem estímulos também é importante (Narzisi, 2020).

Em relação ao Brasil, um artigo publicado por pesquisadores da Universidade Federal de São Carlos (UFSCar) sugere que, para a população com TEA, a dificuldade de compreender o cenário pandêmico e todas as suas ramificações, como as medidas de controle e proteção, a torna mais vulnerável à doença (Fernandes et al., 2020). Essa instituição está pondo em prática um projeto de extensão universitária, chamado "Estratégias de cuidado em Saúde Mental Infanto-juvenil frente à Pandemia", sendo uma das frentes voltada para crianças e adolescentes com TEA e suas famílias. O trabalho envolve ações como: elaboração de material informativo para a população geral e para essas famílias; criação de canal virtual coletivo para dar apoio e dicas para familiares e cuidadores; e ações para a garantia de direitos das pessoas com TEA. Algumas estratégias propostas são: estabelecimento compartilhado de uma rotina em casa, como algo preventivo contra sobrecarga de tarefas e desorganização emocional; flexibilização responsável, e em forma da lei, do isolamento social, buscando amenizar e/ou evitar o sofrimento gerado por ele (como ocorreu em países como França e Espanha); flexibilização do uso de máscara (no dia 02 de julho de 2020, foi sancionada no Brasil a lei federal $n^{\circ} 14.019$, a qual dispensa o uso obrigatório de máscara para pessoas com TEA e outras deficiências que apresentam dificuldades no uso de forma adequada. Porém, para as situações em que seja viável para a criança ou adolescente aderir, buscar experiências mais lúdicas, interessantes e agradáveis para o uso correto da máscara); intervenções informacionais de suporte; e foco particular na atenção psicossocial da família, o que envolve o cuidado de quem cuida dessas crianças e adolescentes que, na maior parte das vezes, são os pais (a telessaúde é uma boa alternativa para o momento, porém deve-se atentar que nem todos possuem acesso a esses recursos). Ademais, é fundamental a conscientização da comunidade sobre as particularidades do universo dos indivíduos com TEA, em prol de mais empatia, solidariedade, espaços de escuta, acolhimento para as famílias dos indivíduos portadores de TEA (Fernandes et al., 2020).

\section{Limitações}

Este estudo possui algumas limitações. Primeiro, que não se trata de uma revisão sistemática com metanálise, porém como há poucos estudos sobre o tema, optou-se pela revisão narrativa e pela não inclusão de critérios mais rigorosos de seleção. Segundo, que revisa apenas os estudos de línguas latinas e anglosaxônicas, o que possivelmente deixou de fora estudos publicados em países com outros idiomas.

\section{Conclusão}

Levando em conta o impacto da pandemia na saúde mental da população geral, é inegável que há uma demanda de novos estudos e estratégias para atender públicos específicos que tendem a sofrer mais com a nova dinâmica imposta pela quarentena. Pais de indivíduos com TEA tendem a estar mais ansiosos, estressados e depressivos nesse período devido a sobrecargas física e emocional. Com maior embasamento científico é possível planejar um cuidado mais direcionado. A diversidade de fatores sociais, econômicos e culturais envolvidos nessa questão 
requer ações multisetoriais e culturalmente adaptadas. Assim, não apenas profissionais da saúde e pesquisadores da área devem agir e se adaptar, como também entidades públicas, instituições de ensino, grupos de apoio e a sociedade com um todo devem ser mobilizados.

\section{Contribuição dos autores}

Medrado AA contribuiu para construção do projeto, delineamento, análise e interpretação dos dados, redação do artigo, interpretação dos resultados, revisão crítica relevante do conteúdo intelectual do artigo científico. Campo RC, Pondé MP e Siquara GM participaram na concepção, revisão crítica e aprovação final da versão a ser publicada. Os quatro autores são responsáveis por todos os aspectos do trabalho na garantia da exatidão e integridade de qualquer parte da obra.

\section{Conflitos de interesses}

Nenhum conflito financeiro, legal ou político envolvendo terceiros (governo, empresas e fundações privadas, etc.) foi declarado para nenhum aspecto do trabalho submetido (incluindo, mas não se limitando a subvenções e financiamentos, participação em conselho consultivo, desenho de estudo, preparação de manuscrito, análise estatística, etc.).

\section{Referências}

Adhikari, S. P., Meng, S., Wu, Y. J., Mao, Y. P., Ye, R. X., Wang, Q. Z., Sun, C., Sylvia, S., Rozelle, S., Raat, H., \& Zhou, H. (2020). Epidemiology, causes, clinical manifestation and diagnosis, prevention and control of coronavirus disease (COVID-19) during the early outbreak period: a scoping review [Epidemiologia, causas, manifestação clínica e diagnóstico, prevenção e controle da doença coronavírus (COVID-19) durante o período inicial do surto: uma revisão do escopo]. Infectious diseases of poverty, 9(1), 1-12. https:// doi.org/10.1186/s40249-020-00646-X

Aguiar, M. C. M. D., \& Pondé, M. P. (2020). Autismo: impacto do diagnóstico nos pais. Jornal Brasileiro de Psiquiatria, 69(3), 149-55. https://doi.org/10.1590/0047-2085000000276

Alhuzimi, T. (2021). Stress and emotional wellbeing of parents due to change in routine for children with Autism Spectrum Disorder (ASD) at home during COVID-19 pandemic in Saudi Arabia [Estresse e bem-estar emocional dos pais devido à mudança na rotina das crianças com Transtorno do Espectro do Autismo (ASD) em casa durante a pandemia de COVID-19 na Arábia Saudita]. Research in Developmental Disabilities, 108, 103822. https://doi. org/10.1016/j.ridd.2020.103822
American Psychiatric Association. (2014). DSM-5: Manual diagnóstico e estatístico de transtornos mentais. Artmed.

Amorim, R., Catarino, S., Miragaia, P., Ferreras, C., Viana, V., \& Guardiano, M. (2020). The impact of COVID-19 on children with autism spectrum disorder [O impacto da COVID-19 nas crianças com transtorno do espectro do autism]. Revista de neurologia, 71(8), 285-91. https://doi. org/10.33588/rn.7108.2020381

Asbury, K., Fox, L., Deniz, E., Code, A., \& Toseeb, U. (2020). How is COVID-19 affecting the mental health of children with special educational needs and disabilities and their families? [Como a COVID-19 está afetando a saúde mental de crianças com necessidades educacionais especiais e deficiências e suas famílias]. Journal of autism and developmental disorders, 51(5), 1772-1780. https://doi. org/10.1007/s10803-020-04577-2

Cahapay, M. B. (2020). How Filipino parents home educate their children with autism during COVID-19 period [Como os pais filipinos educam seus filhos com autismo durante o período da COVID-19]. International Journal of Developmental Disabilities, 1-4. https://doi.org/10.1080/204 73869.2020.1780554

Chen, S. Q., Chen, S. D., Li, X. K., \& Ren, J. (2020). Mental Health of Parents of Special Needs Children in China during the COVID-19 Pandemic [Saúde mental de pais de crianças com necessidades especiais na China durante a Pandemia da COVID-19]. International journal of environmental research and public health, 17(24), 9519. https://doi. org/10.3390/ijerph17249519

Colizzi, M., Sironi, E., Antonini, F., Ciceri, M. L., Bovo, C., \& Zoccante, L. (2020). Psychosocial and Behavioral Impact of COVID-19 in Autism Spectrum Disorder: An Online Parent Survey [Impacto Psicossocial e Comportamental da COVID-19 no Transtorno do Espectro do Autismo: Uma pesquisa online com os pais]. Brain Sciences, 10(6), 341. https://dx.doi. org/10.3390\%2Fbrainsci10060341

DePape, A. M., \& Lindsay, S. (2015). Parents' experiences of caring for a child with autism spectrum disorder [Experiências dos pais em cuidar de uma criança com transtorno do espectro do autismo]. Qualitative health research, 25(4), 569-583. https://doi.org/10.1177/1049732314552455

Dey, M., Castro, R. P., Haug, S., \& Schaub, M. P. (2019). Quality of life of parents of mentally-ill children: a systematic review and meta-analysis [Qualidade de vida dos pais de crianças com problemas mentais: uma revisão sistemática e uma meta-análise]. Epidemiology and psychiatric sciences, 28(5), 563-577. https://doi.org/10.1017/s2045796018000409 
Dhiman, S., Sahu, P. K., Reed, W. R., Ganesh, G. S., Goyal, R. K., \& Jain, S. (2020). Impact of COVID-19 outbreak on mental health and perceived strain among caregivers tending children with special needs [Impacto do surto de COVID-19 na saúde mental e tensão percebida entre os cuidadores que cuidam de crianças com necessidades especiais]. Research in Developmental Disabilities, 107, 103790. https://doi.org/10.1016/j.ridd.2020.103790

Dubey, S., Biswas, P., Ghosh, R., Chatterjee, S., Dubey, M. J., Chatterjee, S., Lahiri, D., \& Lavie, C. J. (2020). Psychosocial impact of COVID-19 [Impacto psicossocial da COVID-19]. Diabetes \& metabolic syndrome, 14(5), 779-788. https://doi. org/10.1016/j.dsx.2020.05.035

Fernandes, A. D. S. A., Speranza, M., Mazak, M. S. R., Gasparini, D. A., \& Cid, M. F. B. (2020). Desafios cotidianos e possibilidades de cuidado às crianças e adolescentes com Transtorno do Espectro Autista (TEA) frente à COVID-19. Cadernos Brasileiros de Terapia Ocupacional, 29, e2121, https://doi.org/10.1590/2526-8910.ctoAR2121

Frye, L. (2016). Fathers' experience with autism spectrum disorder: Nursing implications [A experiência dos pais com a desordem do espectro do autismo: Implicações de enfermagem]. Journal of pediatric health care, 30(5), 453463. https://doi.org/10.1016/j.pedhc.2015.10.012

Garritty, C., Gartlehner, G., Nussbaumer-Streit, B., King, V. J., Hamel, C., Kamel, C., Affengruber, L., \& Stevens, A. (2021). Cochrane Rapid Reviews Methods Group offers evidenceinformed guidance to conduct rapid reviews [O Grupo de Métodos Cochrane Rapid Reviews oferece orientação fundamentada em evidências para conduzir revisões rápidas]. Journal of clinical epidemiology, 130, 13-22. https://doi.org/10.1016/j.jclinepi.2020.10.007

Gomes, P. T., Lima, L. H., Bueno, M. K., Araújo, L. A., \& Souza, N. M. (2015). Autismo no Brasil, desafios familiares e estratégias de superação: revisão sistemática. Jornal de Pediatria (Versão em Português), 91(2), 111-121. https://doi. org/10.1016/j.jped.2014.08.009

Herrema, R., Garland, D., Osborne, M., Freeston, M., Honey, E., \& Rodgers, J. (2017). Mental wellbeing of family members of autistic adults [Bem-estar mental dos membros da família de adultos autistas]. Journal of autism and developmental disorders, 47(11), 3589-3599. https://dx.doi. org/10.1007\%2Fs10803-017-3269-z

Holmes, E. A., O'Connor, R. C., Perry, V. H., Tracey, I., Wessely, S., Arseneault, L., Ballard, C., Christensen, H., Cohen Silver, R., Everall, I., Ford, T., John, A., Kabir, T., King, K., Madan, I., Michie, S., Przybylski, A. K., Shafran, R., Sweeney, A.,... Bullmore, E. (2020). Multidisciplinary research priorities for the COVID-19 pandemic: a call for action for mental health science [Prioridades de pesquisa multidisciplinar para a pandemia da COVID-19: um apelo à ação para a ciência da saúde mental]. The lancet. Psychiatry, 7(6), 547-560. https:// doi.org/10.1016/S2215-0366(20)30168-1
Lee, V., Albaum, C., Tablon Modica, P., Ahmad, F., Gorter, J. W., Khanlou, N., McMorris, Lai J., C., Harrison, C., Hedley, T., Johnston, P., Putterman, C., Spoelstra, M., \& Weiss, J. A. (2020). Impact of COVID-19 on the Mental Health and Wellbeing of Caregivers and Families of Autistic people [Impacto da COVID-19 na saúde mental e bem-estar de cuidadores e famílias de Autistas]. Canadian Institutes of Health Research. https://covid19mentalhealthresearch. ca/wp-content/uploads/2020/06/WEISS-Initial-KnowledgeSynthesis-Report 2020-06-22.pdf

Machado Junior, S. B., Celestino, M. I. O., Serra, J. P. C., Caron, J., \& Pondé, M. P. (2016). Risk and protective factors for symptoms of anxiety and depression in parents of children with autism spectrum disorder [Fatores de risco e proteção para sintomas de ansiedade e depressão em pais de crianças com transtorno do espectro do autism]. Developmental neurorehabilitation, 19(3), 146-153. https:// doi.org/10.3109/17518423.2014.925519

Mutluer, T., Doenyas, C., \& Genc, H. A. (2020). Behavioral Implications of the Covid-19 Process for Autism Spectrum Disorder, and Individuals' Comprehension of and Reactions to the Pandemic Conditions [Implicações Comportamentais do Processo Covid-19 para o Transtorno do Espectro do Autismo, e Compreensão e Reações dos Indivíduos às Condições Pandêmicas]. Frontiers in psychiatry, 11, 561882. https://doi.org/10.3389/ fpsyt.2020.561882

Narzisi, A. (2020). Handle the autism spectrum condition during Coronavirus (COVID-19) stay at home period: Ten tips for helping parents and caregivers of young children [Tratar a condição do espectro do autismo durante o período de permanência do Coronavirus (COVID-19) em casa: Dez dicas para ajudar pais e cuidadores de crianças pequenas]. Brain sciences, 10(4), 207. https://doi. org/10.3390/brainsci10040207

Comitê Científico do Núcleo Ciência pela Infância. (2020). Repercussões da Pandemia de COVID-19 no Desenvolvimento Infantil. NCPI. https://portaldeboaspraticas.iff.fiocruz. br/wp-content/uploads/2020/07/Working-PaperRepercussoes-da-pandemia-no-desenvolvimento-infantil. pdf

Organização Pan-americana de Saúde. (2017). Folha Informativa Transtorno do espectro autista. https://www.paho.org/bra/ index.php?!temid $=1098$

Organização Pan-americana de Saúde. (2020). Folha Informativa sobre COVID-19. https://www.paho.org/bra/index.php?option=com_content\&view=article\&id=6101: covid19\&ltemi$\underline{d}=875 \#$ recomendacoes

Ornell, F., Schuch, J. B., Sordi, A. O., \& Kessler, F. H. P. (2020). Pandemia de medo e COVID-19: impacto na saúde mental e possíveis estratégicas. Revista debates in psychiatry. http://dx.doi.org/10.25118/2236-918X-10-2-2 
Qiu, J., Shen, B., Zhao, M., Wang, Z., Xie, B., \& Xu, Y. (2020). A nationwide survey of psychological distress among Chinese people in the COVID-19 epidemic: implications and policy recommendations [Um levantamento nacional do sofrimento psicológico entre o povo chinês na epidemia da COVID-19: implicações e recomendações políticas]. General psychiatry, 33(2). https://bit.ly/2VP2AMD

Ren, J., Li, X., Chen, S., Chen, S., \& Nie, Y. (2020). The Influence of Factors Such as Parenting Stress and Social Support on the State Anxiety in Parents of Special Needs Children During the COVID-19 Epidemic [A influência de fatores como o estresse dos pais e o apoio social sobre a ansiedade do Estado em pais de crianças com necessidades especiais durante a epidemia da COVID-19]. Frontiers in Psychology, 11, 565393. https://dx.doi. org/10.3389\%2Ffpsyg.2020.565393

Saxena, S. K. (Ed.). (2020). Coronavirus Disease 2019 (COVID-19): Epidemiology, Pathogenesis, Diagnosis, and Therapeutics [Doença do Coronavírus 2019 (COVID-19): Epidemiologia, Patogênese, Diagnóstico e Terapêutica]. Springer Nature. https://www.springer.com/gp/book/9789811548130

Shi, L., Lu, Z. A., Que, J. Y., Huang, X. L., Liu, L., Ran, M. S., Gong, Y. M., Yuan, K., Yan, W., Sun, Y. K., Shi, J., Bao, Y. P., \& Lu, L. (2020). Prevalence of and Risk Factors Associated With Mental Health Symptoms Among the General Population in China During the Coronavirus Disease 2019 Pandemic [Prevalência e fatores de risco associados aos sintomas de saúde mental entre a população geral da China durante a pandemia de Coronavirus de 2019]. JAMA network open, 3(7), e2014053. https://doi.org/10.1001/ jamanetworkopen.2020.14053

Spinelli, M., Lionetti, F., Pastore, M., \& Fasolo, M. (2020). Parents' Stress and Children's Psychological Problems in Families Facing the COVID-19 Outbreak in Italy [O estresse dos pais e os problemas psicológicos dos filhos em famílias que enfrentam o surto da COVID-19 na Itália]. Frontiers in Psychology, 11, 1713. https://dx.doi. org/10.3389\%2Ffpsyg.2020.01713

Sprang, G., \& Silman, M. (2013). Posttraumatic stress disorder in parents and youth after health-related disasters [Transtorno de estresse pós-traumático em pais e jovens após desastres relacionados à saúde]. Disaster medicine and public health preparedness, 7(1), 105-110. https://doi. org/10.1017/dmp.2013.22

Stankovic, M., Jelena, S., Stankovic, M., Shih, A., Stojanovic, A., \& Stankovic, S. (2020). The Serbian Experience of Challenges of Parenting Children with Autism Spectrum Disorders During the COVID-19 Pandemic and the State of Emergency with the Police Lockdown [A experiência sérvia dos desafios dos pais de crianças com transtorno do espectro do autismo durante a pandemia da COVID-19 e o estado de emergência com o fechamento da polícia]. Lancet. http://dx.doi.org/10.2139/ssrn.3582788
Türkoğlu, S., Uçar, H. N., Çetin, F. H., Güler, H. A., \& Tezcan, M. E. (2020). The relationship between chronotype, sleep, and autism symptom severity in children with ASD in COVID-19 home confinement period [A relação entre cronótipo, sono e gravidade dos sintomas do autismo em crianças com ASD no período de confinamento domiciliar COVID-19]. Chronobiology International, 37(8), 1207-1213. https://doi.org/10.1080/07420528.2020.1792485

Ueda, R., Okada, T., Kita, Y., Ozawa, Y., Inoue, H., Shioda, M., Kono, Y., Kono, C., Nakamura, Y., Amemiya, K., Ito, A., Sugiura, N., Matsuoka, Y., Kaiga, C., Kubota, M., \& Ozawa, H. (2021). The quality of life of children with neurodevelopmental disorders and their parents during the Coronavirus disease 19 emergency in Japan [A qualidade de vida das crianças com transtornos neurodisciplinares e de seus pais durante a emergência da doença de Coronavirus 19 no Japão]. Scientific reports, 11(1), 1-8. https://doi. org/10.1038/s41598-021-82743-x

Vasilopoulou, E., \& Nisbet, J. (2016). The quality of life of parents of children with autism spectrum disorder: A systematic review [A qualidade de vida dos pais de crianças com transtorno do espectro do autismo: Uma revisão sistemática]. Research in Autism Spectrum Disorders, 23, 3649. https://doi.org/10.1016/j.rasd.2015.11.008

Xie, S., Karlsson, H., Dalman, C., Widman, L., Rai, D., Gardner, R. M., Magnusson, C., Schendel, D. E., Newschaffer, C. J., \& Lee, B. K. (2019). Family History of Mental and Neurological Disorders and Risk of Autism [História familiar de transtornos mentais e neurológicos e risco de autism]. JAMA network open, 2(3), e190154. https://doi.org/10.1001/ jamanetworkopen.2019.0154

Wang, L., Li, D., Pan, S., Zhai, J., Xia, W., Sun, C., \& Zou, M. (2021). The relationship between 2019-nCoV and psychological distress among parents of children with autism spectrum disorder [A relação entre 2019-nCoV e a angústia psicológica entre pais de crianças com transtorno do espectro do autism]. Globalization and Health, 17(1), 23. https://doi.org/10.1186/s12992-021-00674-8

White, L. C., Law, J. K., Daniels, A. M., Toroney, J., Vernoia, B., Xiao, S., SPARK Consortium, Feliciano, P., \& Chung, W. K. (2021). Brief Report: Impact of COVID-19 on Individuals with ASD and Their Caregivers: A Perspective from the SPARK Cohort [Breve relatório: Impacto da COVID-19 nos indivíduos com ASD e seus cuidadores: Uma perspectiva da SPARK Cohort]. Journal of autism and developmental disorders, 1-8. https:// doi.org/10.1007/s10803-020-04816-6

World Health Organization. (2020, mar 19). Considerations for quarantine of individuals in the context of containment for coronavirus disease (COVID-19): interim guidance [Considerações para a quarentena de indivíduos no contexto da contenção da doença coronavírus (COVID-19): orientação interina]. World Health Organization. https:// apps.who.int/iris/handle/10665/331497 